\title{
Anatomic double-bundle anterior cruciate ligament reconstruction, using CT-based navigation and fiducial markers
}

Keiji Tensho, Hiroyuki Kodaira, Gaku Yasuda, Yasuo Yoshimura, Nobuyo Narita, Susumu Morioka, Hiroyuki Kato, Naoto Saito

K. Tensho, H. Kodaira, G. Yasuda, Y. Yoshimura, N. Narita, S. Morioka, H. Kato

Department of Orthopedic Surgery, Shinshu University School of Medicine, Matsumoto, Japan.

Corresponding author

N. Saito

Department of Applied Physical Therapy, Shinshu University School of Health Sciences, Asahi 3-1-1, Matsumoto, Nagano 390-8621, Japan.

e-mail: saitoko@shinshu-u.ac.jp

TEL, FAX: +81 263372409 


\begin{abstract}
Purpose: Accurate placement of separate anteromedial and posterolateral bundle bone tunnels is crucial for anatomic, double-bundle anterior cruciate ligament (ACL) reconstruction. However, identifying the anatomical footprint at which to make the tibial and femoral bone tunnels is not a straightforward procedure. To overcome this problem, we used a CT-based navigation technique with a registration procedure based on fiducial markers (FMs).
\end{abstract}

Methods: Preoperatively, 10 FM points were placed on skin around knee joint and scanned with CT. Imaging data of the knee were recorded on the computer system for preoperative registration and surgical planning. Intraoperatively, with a reference frame fixed to the distal medial aspect of femur and tibia, paired-point matching registration was performed with use of points marked on skin through FM center holes. During tibial tunnel guide wire placement, tibial aiming guide with tracking device fed back the position of tip and direction of the guide wire on the three-dimensional (3D) tibia bone surface image and multiple image planes in real-time. For the femoral side, the navigation pointer was placed at the footprint center with visual guidance of $3 \mathrm{D}$ image of lateral wall sagittal view on navigation monitor and marked with navigation awl.

Results: The average registration accuracy of 22 consecutive patients was $0.7 \pm 0.2 \mathrm{~mm}$ and $0.6 \pm 0.2 \mathrm{~mm}$ for femoral and tibial bone, respectively. Most of the bone tunnel positions evaluated with 3D-CT image were confirmed to be accurately placed in reference to the preoperative plan. There was no damage to femoral condyle cartilage and no other complication.

Conclusion: This new CT-based computer navigation system opens the possibility for 
surgeons to plan bone tunnel positioning preoperatively and control it during technically demanding anatomic double-bundle ACL reconstruction.

\section{Key words}

ACL reconstruction, CT, Navigation, Fiducial marker 


\section{Introduction}

Currently, anterior cruciate ligament (ACL) reconstruction is the standard operation for ACL injury in active patients. However, long-term clinical studies reveal unsatisfactory postoperative results and concern regarding the conventional technique of ACL reconstruction in terms of residual laxity, especially in controlling rotation has been expressed [2]. Recently, a large research effort has been underway to fully define the functional roles, insertion site anatomy, and biomechanics of the 2 ACL bundles $[4,7,9,11,13]$, and clinical studies have shown that more anatomical approaches resulted in superior outcome when compared to single-bundle ACL reconstruction $[1,14]$.

However, an anatomical approach with separate reconstruction of both ACL bundles is more technically demanding and time-consuming than the single-bundle procedure. In particular, the proper creation of graft insertion sites remains a difficult point and a key objective. Because subjective results of existing methods using conventional tools are not as satisfactory as desired even for single-bundle procedure [5, 10], a larger number of failures in bone tunnel preparation should be expected in double-bundle reconstruction.

Recently there have been many reports on ACL surgery using several different computer assisted systems (CAS) to improve the reproducibility of graft placement $[3,6]$. These systems include information on the distance from the over-the-top position, the clockwise position, and isometry deviations during knee motion. Ferretti et al. and Purnell et al. reported bony landmarks at the ligament-bone junction [4,9], and procedures that use these landmarks as reference are becoming popular. Thus, to fully utilize the current knowledge of osseous landmarks of the femoral and tibial attachments, a virtual representation of the original 3D bone surface of tibia and femur by navigation guide system was needed. We hypothesized that CT-based navigation 
offers a possibility to achieve accurate bone tunnel preparation. As far as we could find, there has been no report on such a procedure using CT-based navigation. We developed a new CT-based navigation technique with a registration procedure based on skin fiducial markers (FMs).

\section{Materials and Methods}

A surgical navigation system (StealthStation Treon plus; SofamorDanek, Memphis, TN), consisting primarily of a computer system, a charge-coupled device camera, and the bundled spine application was used for the anatomical reconstruction of ACL.

For the registration procedure in this study, FMs (SofamorDanek, Memphis, TN) (Fig. 1a) were used. These markers were donut-shaped and obvious on CT scans, and it was possible to mark on the skin through the central hole. On the pre-operative day, FMs were placed randomly at ten points around the knee joint in three dimensions before CT (Fig. 1b). The patients then underwent CT scans with the neutral hip and extended knee positions as relaxed as possible. Basic imaging data used for navigation (slice thickness, $1 \mathrm{~mm}$ ) of the knee were recorded on the system computer for pre-registration and surgical planning. On the navigation computer, three 3D images (knee, proximal tibia, lateral half of femoral condyle) were reconstructed. The 3D image of knee was used for pre-registration of FMs. The centers of the FMs at each of the ten points were pointed to and recorded, manually (Fig. 2a). The 3D image and sagittal and coronal image sections of proximal tibia were used for planning of tibial tunnel position and direction. The direction of the tibial tunnel for the anteromedial (AM) bundle was planned at 30-35 degrees from the tibial shaft axis in the coronal plane and 40-45 degrees in the sagittal plane, and for the posterolateral (PL) bundle at 40-45 degrees in the coronal plane and 35-40 degrees in the sagittal plane. Outlets of tunnels were set at 
the anatomical insertion site of each bundle (Fig. 2b).

For the creation of femoral tunnels, the 3D image of lateral half of femoral condyle was reconstructed on a monitor for better view of lateral wall and roof of the femoral intercondylar notch. A transtibial or accessory anterior medial portal technique was used for creation of femoral tunnel, and the target was set on the center of the anatomical footprint of each bundle in reference to intercondylar ridge and bifurcate ridge if it was identified (Fig. 2c). The 2 intra-articular outlets of the femoral tunnels did not overlap on the lateral femoral condyle in any of the knees encountered.

Before surgery, skin marking was done through the center hole of each FM. After the removal of markers and skin disinfection, reference frames were fixed percutaneously to the distal tibia diaphysis and medial aspect of distal femur, each with two 2.5-mm half pins. We used three navigation guides: a passive navigation pointer, tibial aiming guide, and navigation awl (Fig. 3a,b). The tibial aiming device was a commercially available tibial drilling guide, equipped with a directly mounted navigation array. The navigation awl was used for marking of femoral footprint. After setting navigation guides, registration was started. Registration was performed using paired-point matching: 10 points around the knee were registered with use of the navigation pointer (Fig. 3c). During the registration, attention should be paid to reproduce the leg position during CT imaging with the hip in neutral position and knee in extended position. When the predicted registration accuracy was more than $1.0 \mathrm{~mm}$, repeated paired matching registration was needed until an accuracy of less than $1.0 \mathrm{~mm}$ was obtained. After successful registration, graft harvesting and diagnostic arthroscopy was performed.

The tip of the tibial aiming guide was placed at the center of the AM and PL bundle at the tibial insertion site, and tip placement and direction of aimer were projected onto 
the navigation monitor and adjusted for pre-operative planning. After the aiming device was set, each guide wire was drilled through the sleeve. The position of guide wire tip on the tibial plateau was checked with the navigation pointer to compensate for any deflection of guide wire.

To create the femoral tunnel, a 3D lateral image of distal femur sagittal view was shown on the navigation computer monitor. The femoral footprints of the AM bundle were marked using the navigation awl with the assistance of the computer monitor images projected during pre-operative planning. Transtibial drilling through each tibial tunnel was our first option. However, if the femoral marking holes were hard to reach using the transtibial approach, we created femoral tunnels through the accessory anteromedial portal. When we selected the anterior medial portal approach, simulation was done before inserting the guide wire. On the navigation computer screen, the 3D image of the distal femur was rotated to face the posterolateral aspect of the femur. The virtual femoral tunnel diameter was set to the real diameter of the AM graft. The navigation pointer tip was placed at a small hole on the AM footprint, and knee was flexed 90 to 120-130 degrees. When flexing the knee, risk of the posterior wall breakage and sufficiency of the bone tunnel length was evaluated (Fig. 4).

We retrospectively reviewed 22 consecutive patients who had undergone anatomical ACL reconstruction using this technique and evaluated intraoperative registration accuracy, the deviation of the actual bone tunnel position compared to the planned position by 3D-CT postoperatively, and the presence of complication.

\section{Results}

The average registration accuracy was $0.7 \pm 0.2 \mathrm{~mm}(0.2-1.1 \mathrm{~mm})$ and $0.6 \pm 0.2 \mathrm{~mm}$ (0.3-1.1 mm) for femoral and tibial bone, respectively. Registration accuracy of $1 \mathrm{~mm}$ 
or less was not achieved in only 2 patients ( 1 tibial, 1 femoral side). In 2 patients in femoral side, bone tunnel was prepared following conventional methods, because the navigation system could not be used as the deviation of frame position resulted in decreased accuracy.

Bone tunnel positions were postoperatively evaluated from 3D-CT images, and most of the bone tunnel positions were confirmed to be accurately placed in reference to the preoperative plan (Fig. 5). There was no case of injury to femoral condyle cartilage, artery, or nerve, and no sign of infection.

\section{Discussion}

The most important finding of the present study was that anatomical ACL reconstruction using CT-based navigation was practically acceptable. CAS benefits surgeons by providing feedback on the positions of instruments and bony anatomy inside the surgical field. Among various navigation procedures for ACL reconstruction reported $[3,6]$, we believe that the CT image-based procedure reported here is the most suitable for the current surgical technique and has various advantages.

As the first major advantage, preoperative planning based on 3D images of patient's bone is possible. Ferretti et al. reported that osseous landmarks around the footprint on femoral side, including intercondylar ridge or bifurcate ridge, could be used to decide the insertion point [4]. Also, Purnell et al. reported that 3D images reconstructed from high resolution CT data depicted detailed surface bone morphology and confirmed osseous landmarks around the ACL footprints of femur and tibia [9]. In our procedure, 3D images were reconstructed from preoperative CT data, and the images of actual bone surface morphology of the patient could be used for planning of bone tunnel direction and outlets on joint surface, and thus bone tunnel planning was 
possible using these landmarks for each patient.

As the second advantage, CT-based navigation is applicable to various surgical techniques and can provide useful information for each procedure during the surgery. The transtibial method for femoral tunnel preparation is limited to reach the anatomical femoral footprint, because with a narrower tibial tunnel diameter compared to the single bundle procedure, the influence by tunnel direction or outlet on tibial condyle becomes greater. In our procedure, this kind of mismatch could be reduced by preoperative planning of direction of tibial bone tunnel and outlet on tibial condyle, and by strict control during the surgery. However, several recent studies have shown that transtibial approach can limit the ability to place the femoral tunnel anatomically, even if tibial tunnels are placed in accurate positions. For this reason, the AM portal procedure has been increasingly reported recently. On the other hand, in this procedure, risk of cartilage injury and posterior wall breakage of lateral femoral condyle had been reported [15]. In the presently reported navigation system, pertinent information could be obtained using a navigation pointer before actual guide wire insertion, including insertion direction, confirmation of whether injury to cartilage or posterior wall breakage had been avoided, or whether sufficient length of bone tunnel was secured.

As the third advantage, the setting of navigation is simple, requiring only a time as short as $10-15 \mathrm{~min}$. from frame insertion to registration. This step can be done before surgery, thus reducing surgical time.

This study has several limitations, such as small number of cases, and no evaluation of clinical results or accuracy of tunnel positions created using this system. To resolve these limitations, we are now accumulating cases and investigating accuracy and reproducibility by comparing preoperative plans to postoperative bone tunnel position using $3 \mathrm{D}-\mathrm{CT}$ in accordance with the 
method of Tsuda et al [12]. The preliminary results as the initial step support its usefulness.

\section{Conclusion}

This technique could reduce the burden on surgeons by intraoperative image assistance and is highly beneficial. Recently, ACL reconstruction has become increasingly complicated and technically demanding. The navigation system reported here is applicable to current surgical techniques and is a useful procedure capable of supporting arthroscopic surgery.

\section{Conflict of interest statement}

The authors report no conflict of interest. 


\section{References}

1. Aglietti P, Giron F, Losco M, Cuomo P, Ciardullo A, Mondanelli N (2009) Comparison between single- and double-bundle anterior cruciate ligament reconstruction: a prospective, randomized, single-blinded clinical trial. Am J Sports Med 38:25-34

2. Biau DJ, Tournoux C, Katsahian S, Schranz P, Nizard R (2007) ACL reconstruction: a meta-analysis of functional scores. Clin Orthop Relat Res 458:180-187

3. Colombet PD, Robinson JR (2008) Computer-assisted, anatomic, double-bundle anterior cruciate ligament reconstruction. Arthroscopy 24:1152-1160

4. Ferretti M, Ekdahl M, Shen W, Fu FH (2007) Osseous landmarks of the femoral attachment of the anterior cruciate ligament: an anatomic study. Arthroscopy 23:1218-1225

5. Harner CD, Giffin JR, Dunteman RC, Annunziata CC, Friedman MJ.(2001) Evaluation and treatment of recurrent instability after anterior cruciate ligament reconstruction.Instr Course Lect.50:463-474

6. Hart R, Krejzla J, Sváb P, Kocis J, Stipcák V (2008) Outcomes after conventional versus computer-navigated anterior cruciate ligament reconstruction. Arthroscopy 24:569-578

7. Petersen W, Zantop T (2006) Anatomy of the anterior cruciate ligament with regard to its two bundles. Clin Orthop Relat Res 454:35-47

8. Pombo MW, Shen W, Fu FH(2008) Anatomic double-bundle anterior cruciate ligament reconstruction: where are we today?Arthroscopy 24:1168-1177

9. Purnell ML, Larson AI, Clancy W (2008) Anterior cruciate ligament insertions on the tibia and femur and their relationships to critical bony landmarks using 
high-resolution volume-rendering computed tomography. Am J Sports Med 36:2083-2090

10. Sudhahar TA, Glasgow MM, Donell ST.(2004) Comparison of expected vs. actual tunnel position in anterior cruciate ligament reconstruction. Knee 11:15-18

11. Takahashi M, Doi M, Abe M, Suzuki D, Nagano A (2006) Anatomical study of the femoral and tibial insertions of the anteromedial and posterolateral bundles of human anterior cruciate ligament. Am J Sports Med 34:787-792

12. Tsuda E, Ishibashi Y, Fukuda A, Yamamoto Y, Tsukada H, Ono S.(2010) Tunnel position and relationship to postoperative knee laxity after double-bundle anterior cruciate ligament reconstruction with a transtibial technique. Am J Sports Med. 38:698-706

13. Yagi M, Wong EK, Kanamori A, Debski RE, Fu FH, Woo SL (2002) Biomechanical analysis of an anatomic anterior cruciate ligament reconstruction. Am J Sports Med 30:660-666

14. Yasuda K, Kondo E, Ichiyama H, Tanabe Y, Tohyama H (2006) Clinical evaluation of anatomic double-bundle anterior cruciate ligament reconstruction procedure using hamstring tendon grafts: comparisons among 3 different procedures. Arthroscopy 22:240-251

15. Zantop T, Haase AK, Fu FH, Petersen W (2008) Potential risk of cartilage damage in double bundle ACL reconstruction: impact of knee flexion angle and portal location on the femoral PL bundle tunnel. Arch Orthop Trauma Surg 128:509-513 


\section{Figure legends}

Figure 1

(a) FM is donut-shaped, and the skin can be marked through the central hole.

(b) FMs were patched at ten points around knee joint before performing CT scan.

Figure 2

(a) Preregistration was performed by registering the points in the computer.

Center of the marker on the preoperative CT image in the computer monitor was manually pointed.

(b) Tibial side: before operation, exit of each bundle bone tunnel was planned by 3D image of CT and direction of tunnel was planned by coronal and sagittal image. Diameter of the plan was set at that of virtual tibial bone tunnel. (Green: plan of the AM bundle, yellow: plan of the PL bundle; diameter of the plan was set at $7.0 \mathrm{~mm}$ )

(c) Femoral side: 3D view of lateral wall and roof of femoral intercondylar notch was used for planning and the footprint of each fiber bundle was set as an insertion point. Osseous landmarks (black arrow: intercondylar ridge, white arrow: bifurcate ridge) were also used as reference when they could be identified. (Pink: insertion point of AM bundle, blue: insertion point of PL bundle)

Figure 3

(a) Tibial aiming guide equipped with tracking device was used to place guide wire in a position of preoperative planning of tibial tunnel.

(b) Navigation awl was used for marking on the bone surface of femoral footprint.

(c) Intraoperative registration was performed by registering the points marked on the skin using the navigation pointer. 


\section{Figure 4}

Diameter of the virtual femoral tunnel is set at the actual graft diameter, and a small hole made by awl is pointed by the passive navigation pointer. Using the trace, risk of lateral femoral condyle injury and posterior wall breakage, and bone tunnel length are evaluated.

(a) Knee joint bent 90 degrees: virtual femoral tunnel revealed risk of femoral condyle injury and insufficient bone tunnel length.

(b) Knee joint bent 120 degree: risk of femoral condyle injury was avoided by bending the knee joint and sufficient bone tunnel length was secured.

\section{Figure 5}

Pre- and post-operative 3D images.

(a, b) Preoperative plan. (Femoral side, Pink: insertion point of AM bundle, blue:

insertion point of PL bundle; Tibial side, Green: plan of the AM bundle, yellow: plan of the PL bundle)

(c, d) Post-operative image showing that 2 tunnels were created at the planned position. 


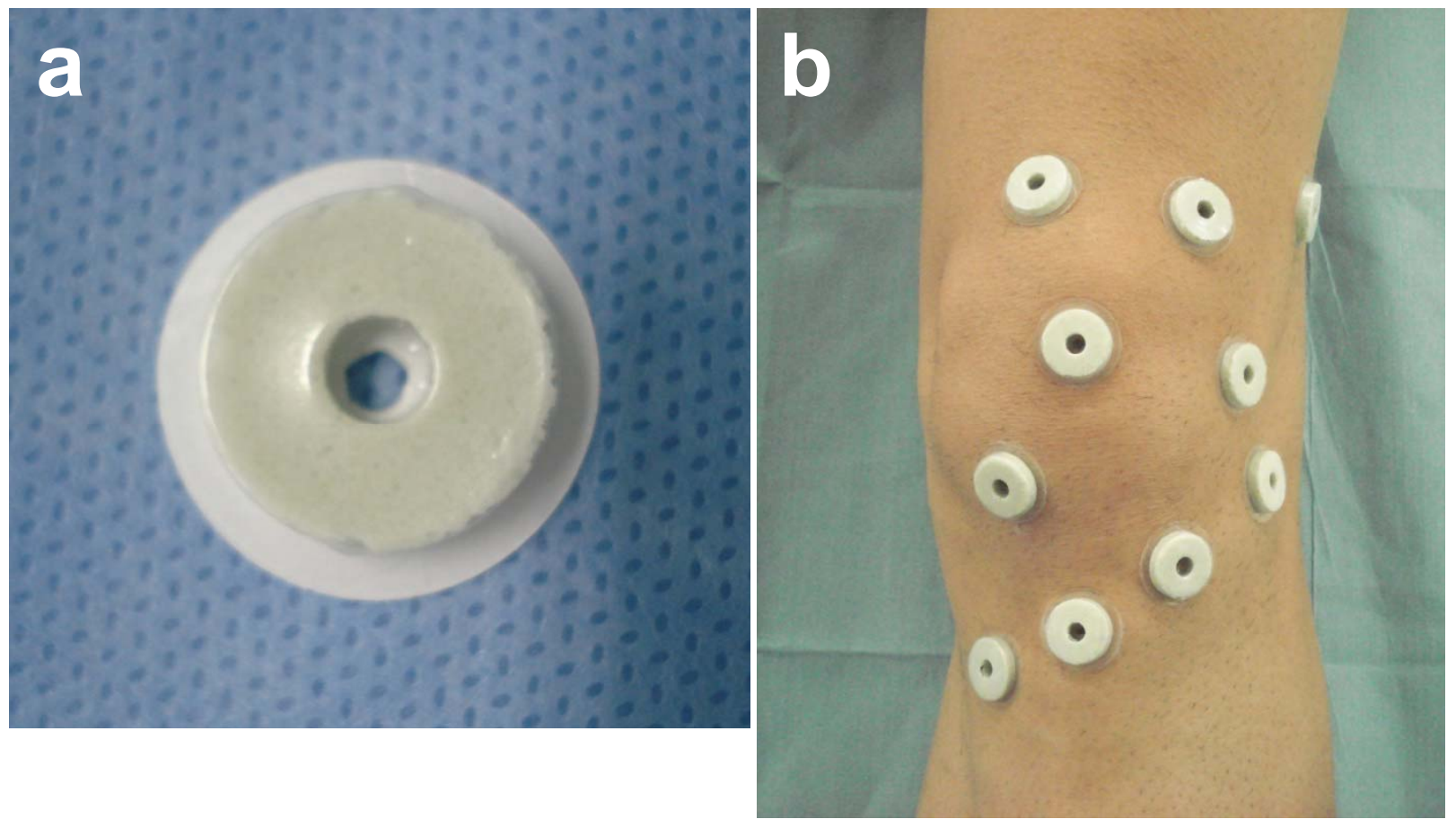

Fig. 1 


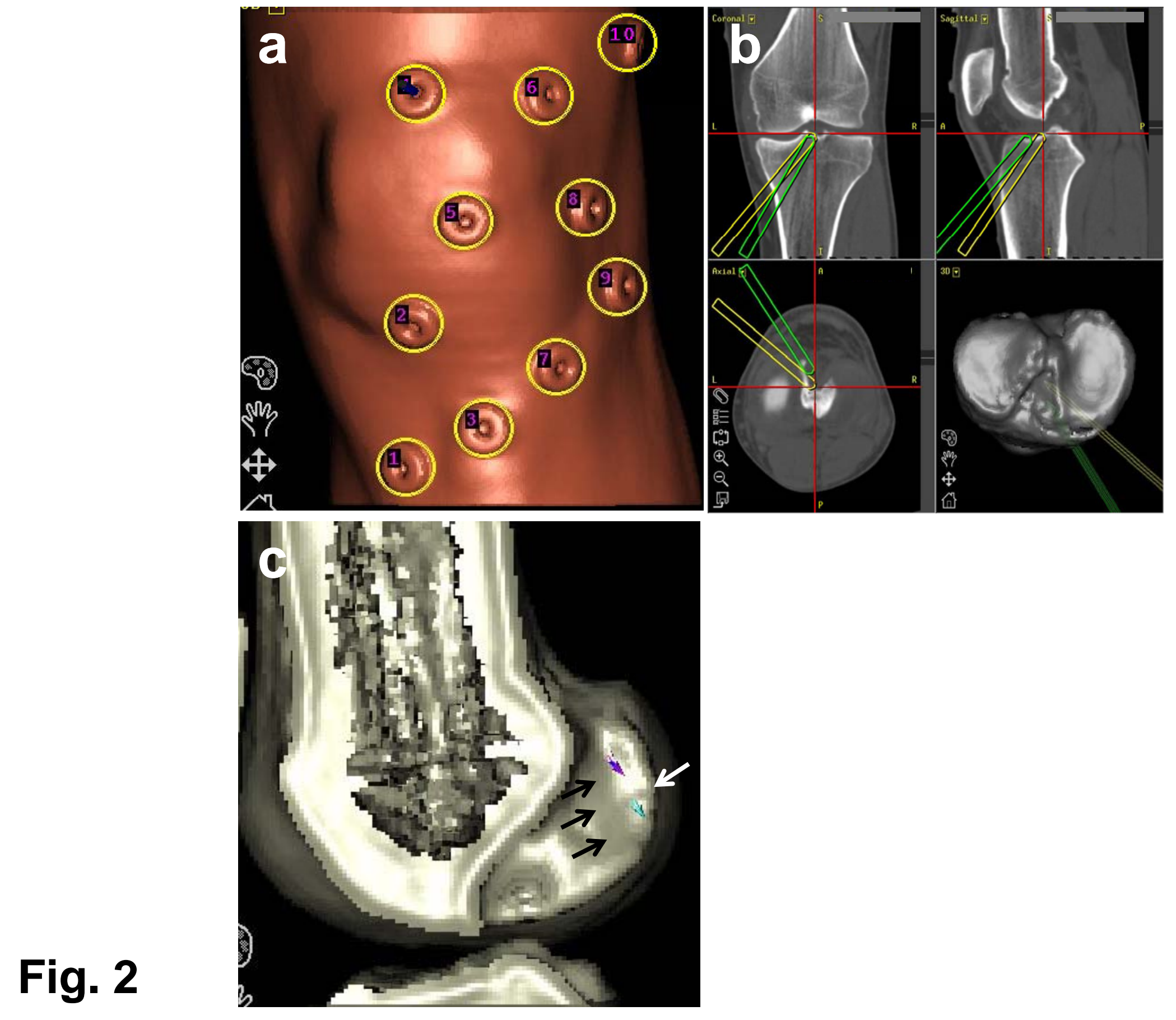




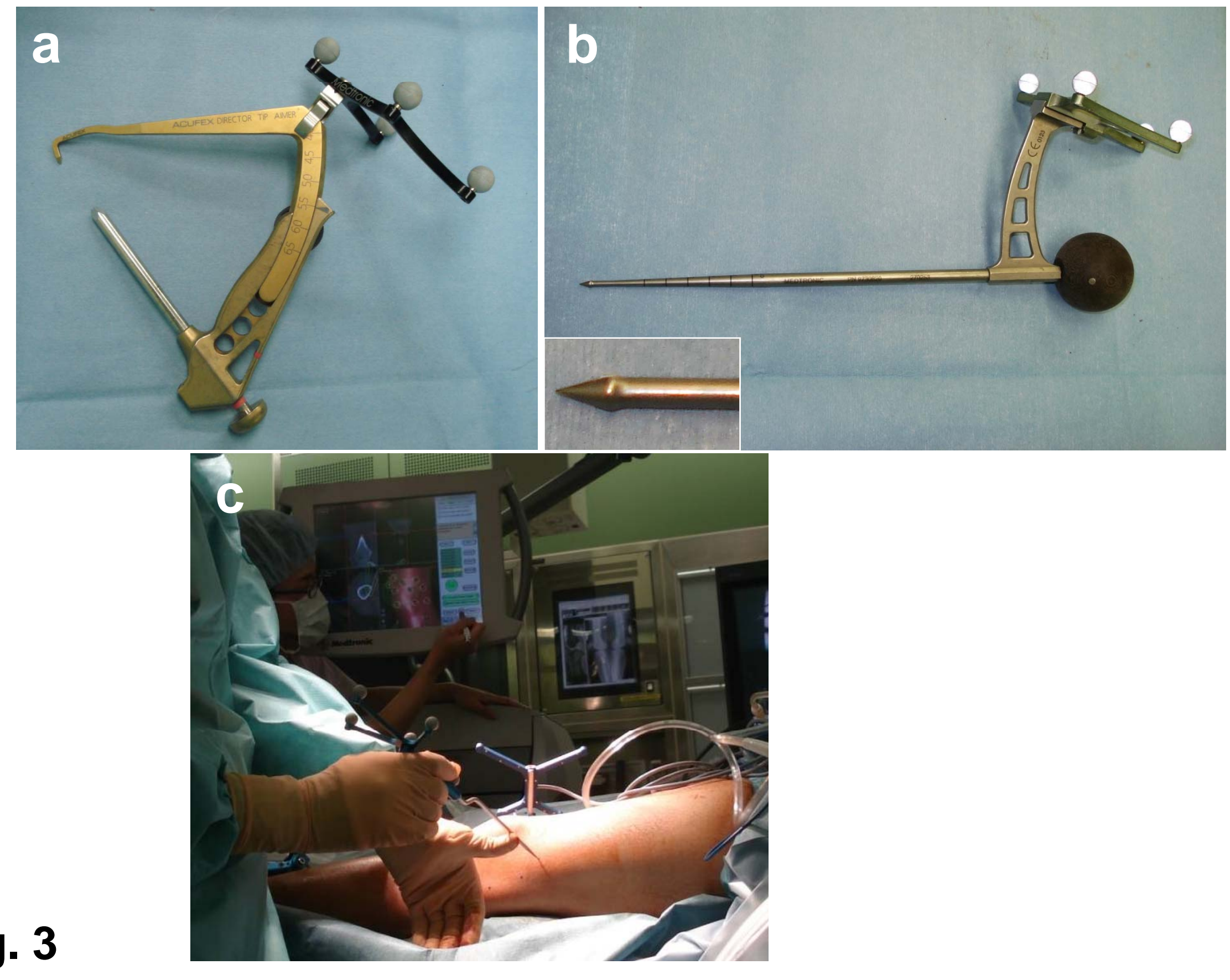

Fig. 3 


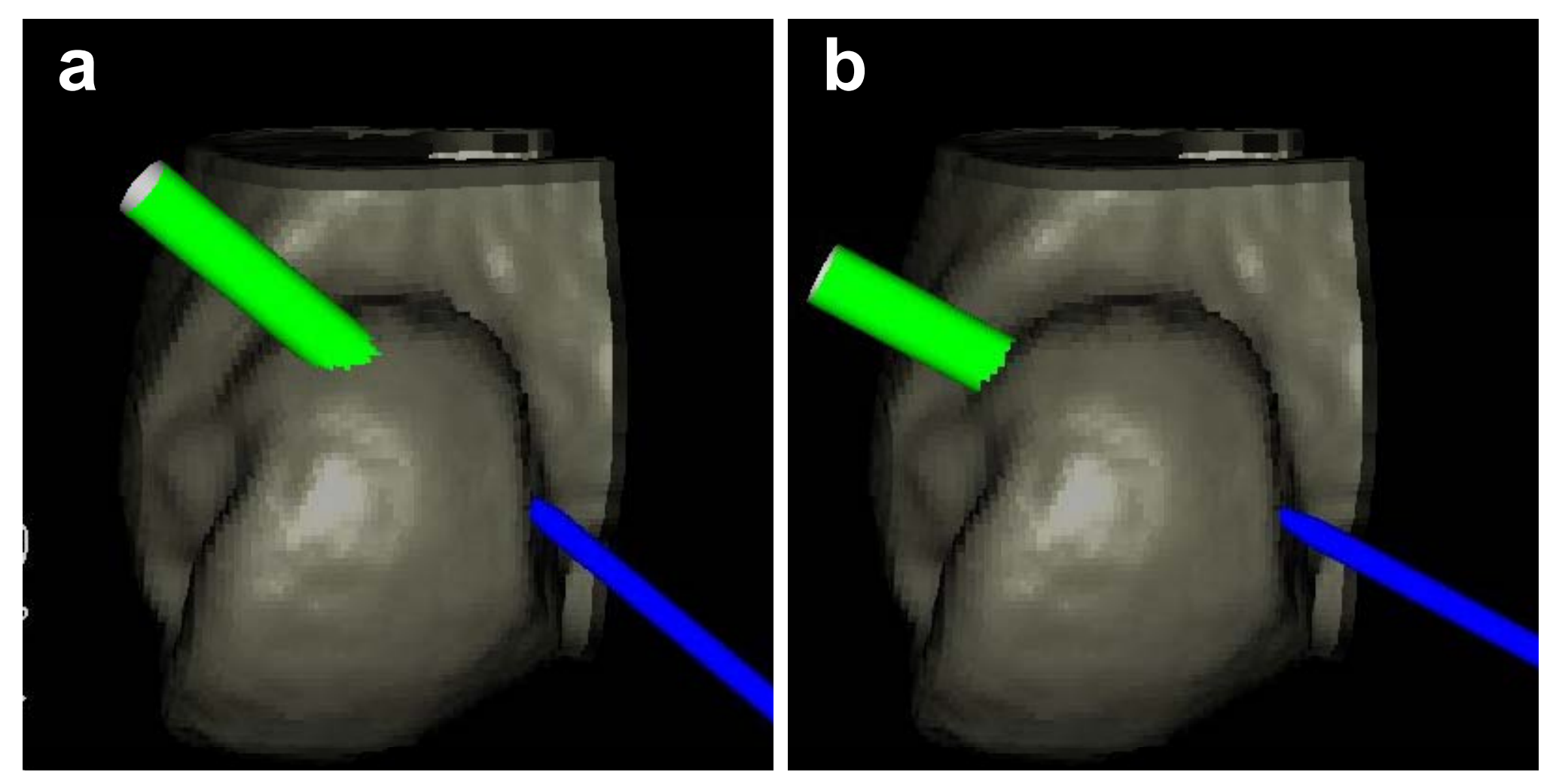

Fig. 4 


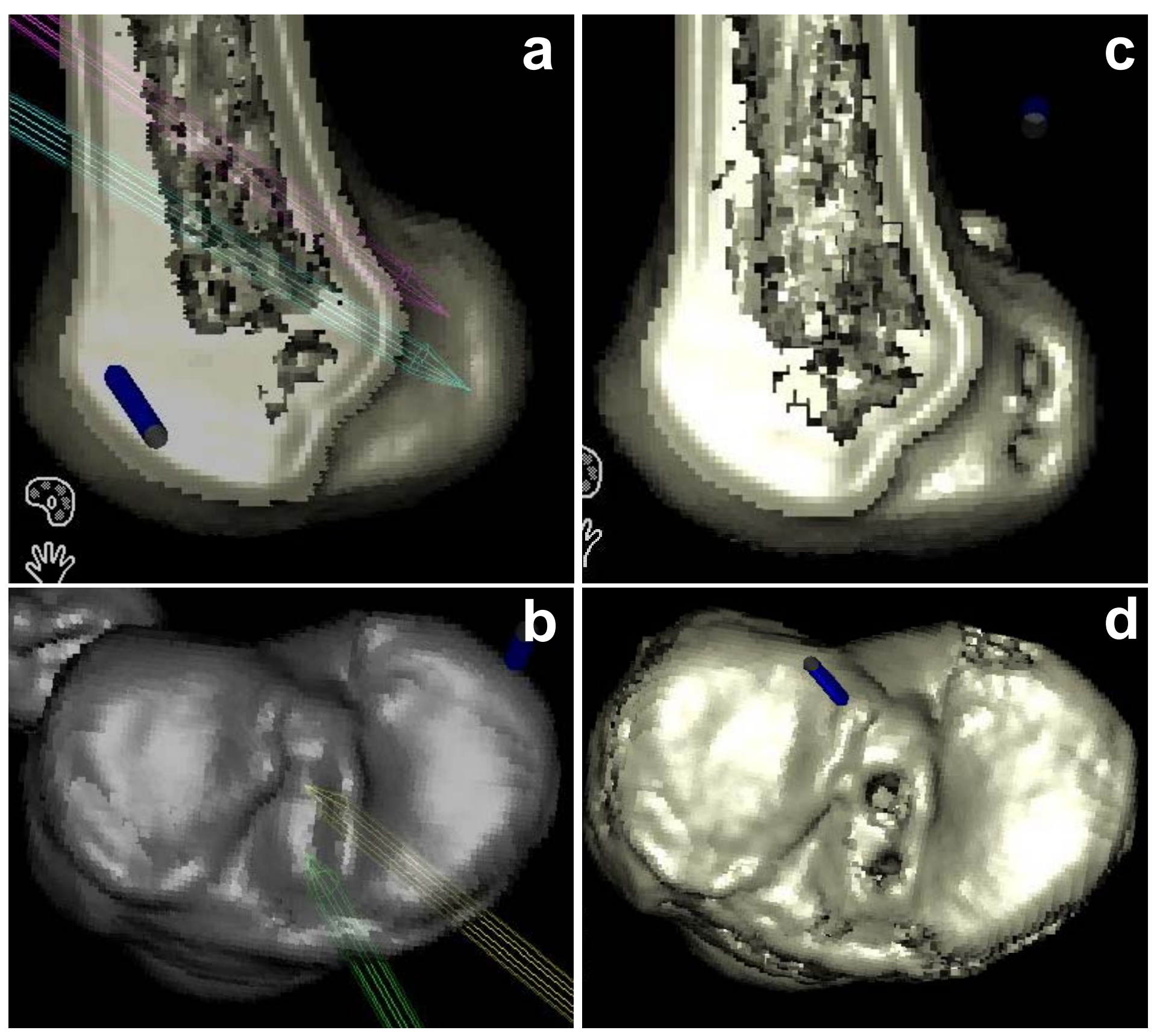

Fig. 5 